\title{
Implementation of evidence-based practice by standardized care plans: A study protocol
}

\author{
Inger Jansson $^{1^{*}}$, Eva Törnvall ${ }^{2}$ \\ ${ }^{1}$ School of Social and Health Sciences, Halmstad University, Halmstad, Sweden \\ ${ }^{2}$ Research and Development Unit, Local Health Care, County of Ostergotland, Linköping, Sweden \\ Email: *inger.jansson@hh.se
}

Received 7 October 2013; revised 9 November 2013; accepted 28 November 2013

Copyright (c) 2013 Inger Jansson, Eva Törnvall. This is an open access article distributed under the Creative Commons Attribution License, which permits unrestricted use, distribution, and reproduction in any medium, provided the original work is properly cited.

\begin{abstract}
Background: Patient records should both transfer and create knowledge about patients and their health care. A standardized care plan could be a way to implement evidence-based care directly in practice and improve the documentation in patient records. The aim of this study is to investigate and compare the development and implementation process of a standardized care plan in hospital and primary health care. A further aim is to evaluate the effects on the quality of documentation and the care given in two contexts. Methods and Analysis: Realistic evaluation will be used as a framework to investigate the implementation process. According to this framework, possible contexts, mechanisms, and outcomes in the study will be considered. The study will be performed in two contexts: an orthopedic clinic and primary health care centers. In both contexts, the two key mechanisms will be the same: the implementation process will be driven by internal facilitators (practitioners at the units) and the process will be guided by the Rules and Regulations for interoperability in the Health and Social Care specification, "National information structure for standardized care plans". Two outcomes of the study will be studied: to investigate the development and implementation process by an evaluation of fidelity and to evaluate how a standardized care plan affects the quality of documentation and the use of evidence-based care. Discussion: Implementation of the SCP will probably meet the same resistance as implementation of guidelines. Documentation of care is an important but resource-consuming requirement in health care, a more standardized method of documenting is requested by health professionals. This project can provide insight into the complex process of developing and implement an SCP in different

*Corresponding author.
\end{abstract}

contexts, which will be useful in further implementation processes.

Keywords: Standardized Care Plan; Orthopedic Clinic; Primary Health Care; Realistic Evaluation

\section{INTRODUCTION}

To implement evidence-based knowledge in health care is a responsibility and a challenge [1-3]. The goal of evidence-based care is to use the methods that do the most good. One way to implement evidence-based practice is by clinical guidelines [4], which provide evidence-based generic recommendations for best practice for a specific clinical domain $[5,6]$. The use of guidelines also means more opportunity for equal and comparable care [7]. Unfortunately, adherence to guidelines varies and sometimes different guidelines for the same clinical domain are used at the same clinic [7]. Reasons for the defective use of guidelines can be poor knowledge, lack of information, lack of personal experience, lack of feedback, lack of leadership, and/or lack of a culture to improve care [7].

Health care is an information-intensive activity and the patient record is one of the most important sources and tools for information management. The patient record should both transfer and create knowledge about the patient and their health care [8]. To meet these requirements, the documentation in the patient record must be systematic and rigorous, and standardized structures, terminologies, and classification systems must be used [8]. Earlier studies of nurses' documentation showed deficiencies concerning data about, for example, nursing diagnosis and outcomes [9].

A standardized care plan (SCP) could be a way to implement evidence-based care directly in practice and improve the documentation in the patient records. In Sweden, the term SCP is used to describe a plan of health 
care predefined on the basis of a defined knowledge base, describing recommended interventions for patients with one or more health problems [10]. In the literature, an SCP may be referred as a critical pathway, clinical pathway, care model, care map, evidence-based care guideline, or protocol [11]. In a review, Kinsman, Rotter, James, Snow and Willis [12] suggested the term clinical pathway to identify a structured evidence-based multidisciplinary plan of care. Three of the following four criteria must be fulfilled: the pathway must be used to translate guidelines or evidence into local structures; the pathway should comprise detailed steps for treatment or care; the pathway must have a time line; the pathway should aim to standardize care in one clinical domain [12]. Since SCP is a term used in Sweden this term will be used in this work.

In the Swedish Strategy for eHealth, work is taking place nationally to create and implement a Swedish information structure that is a generic description of the health and social care process encompassing the required information and an interdisciplinary terminology in health care [13]. A general specification defining SCP and the development and implementation of SCP exists in Sweden: Rules and Regulations for Interoperability in Health and Social Care (RIV, acronym in Swedish) [10] and work is ongoing to develop and test SCP around the country $[14,15]$.

Earlier studies have shown that nurses perceive that SCPs increase their ability to provide the same quality of care to all patients and reduce the time spent on documentation as well as unnecessary documentation $[14,16]$. Pöder, Fogelberg-Dahm and Wadensten [17] found that perceived knowledge about evidence-based guidelines increased after implementation of SCP. According to Vanhaecht, De Witte, Panelle and Sermeus [18] clinical pathways support clinical processes and have a positive impact on coordination of care and organization of care processes.

The main factors that motivated nurses to implement SCPs [15] were that they were easy to understand and follow and corresponded to organizational norms. In the implementation process, internal facilitators acted as important educators, providing reminders to use the SCP and feedback to the SCP users. According to Hunter and Segrott [19], clinical pathways seem to achieve effects on clinical care and professional identities at the development stage. The aim of reducing documentation is often mentioned when clinical pathways are introduced.

Despite the regulation and positive effects of using SCP, the use of SCP in Swedish health care is not common and therefore it is useful to investigate the development and implementation process as well as the effects of the process. Contextual factors have a crucial role in how successful an implementation became. Matland [20] describe how conflicts and ambiguity can constitute both hindrance and possibilities for implementation of policies and guidelines. When implementing guidelines one has to accept that there will be conflicts and find a way to solve them. The ambiguity in a guideline gives scope for compromises which can facilitate the acceptance of a guideline. Therefore, it is relevant to study two different contexts and then compare them.

The aim of this study is to investigate and compare the development and implementation process of SCPs in hospital and primary health care. A further aim is to evaluate the effects on the quality of documentation and care given in two different contexts.

\section{METHODS}

\subsection{Theoretical Framework}

There are several different theories about implementation. The theories have different perspectives such as the individual, the context and social interaction, and the organization. For this study, we have chosen realistic evaluation as a framework to investigate the implementation process. Realistic evaluation aims to find out how a program works, for whom and under what circumstances, and can be based on methodological pluralism [21-23]. Realistic evaluation is guided by three themes: to understand the mechanisms through which the intervention produces change; to understand the contextual conditions necessary to trigger these mechanisms; and to develop outcome pattern predictions according to the context and mechanisms triggered [21]. The investigator initially considers possible contexts, mechanisms, and outcomes (CMO) to describe a program and then gathers data. The results are then used to revise the initial CMO propositions. This framework has been described previously in study protocols and research [22,24].

Because the starting point of this study is in real services and involves real health personnel, the causal mechanisms are embedded in the particular contexts and their social processes; realistic evaluation offers a way to describe and understand the complex relationship between mechanisms and the effect the context has on their practicability and outcomes.

\subsection{Implementation Strategy}

According to the realistic evaluation framework [21], initially CMOs in the study are considered (Table 1). The study will be performed in two different contexts (C). One context is an orthopedic clinic at one regional hospital in a county in the south of Sweden and the other context is 11 primary health care centers (PHCC) in another county in the south of Sweden where a university hospital is sited. The differences between the two contexts will be mapped and described. The main differ- 
ences known so far are the types of health services that are offered and the experiences of using SCPs at an orthopedic clinic and a PHCC. At an orthopedic clinic, the patient is admitted for a limited time and around the clock. In a PHCC, the care process extends over unlimited time but is mostly performed during office hours and often, the patient has a high degree of comorbidity. At an orthopedic clinic, the personnel may have experience using SCPs, whereas use of SCPs is infrequent in PHCCs.

At an orthopedic clinic, an SCP for patients with hip fracture will be developed and implemented. In PHCCs, an SCP for patients with leg ulcers and patients with hypertension will be developed and implemented. The intention is to develop a multi-disciplinary SCP.

In both contexts, the two key mechanisms (M) will be the same. The first mechanism is that the process will be driven from a bottom-up perspective, whereby the implementation will be driven by internal facilitators who are practitioners at the units. A facilitator often acts as a change agent for the implementation of the SCP and the facilitator is believed to be central to successful implementation [22]. The second mechanism is that the process will be guided by the RIV specification "National information structure for standardized care plans” [10]. A RIV specification is an established method to describe IT support in a development project in Sweden. In the RIV specification, the development of an SCP is described as natural part of the work to develop care and make the care safer. It is also important that the base for an SCP is scientific, evidence-based, and experience-based knowledge and that the SCP is multi-professional. From the knowledge that the SCP is based on, the goal for health care should be identified as well as the interventions that are relevant.

Project groups of internal facilitators will be established to develop an SCP and implement it within the electronic patient record. At the orthopedic clinic, a multi-professional group will be established and at the

Table 1. Initially CMOs considered in the study, according to the realistic evaluation framework [21].

\begin{tabular}{lll}
\hline Context & Mechanism & Outcome \\
\hline $\begin{array}{l}\text { A: Onthopedic } \\
\text { clinic }\end{array}$ & $\begin{array}{l}\text { responsible for } \\
\text { development and } \\
\text { implementation of the } \\
\text { SCP at the units }\end{array}$ & $\begin{array}{l}\text { Implementation } \\
\text { process: To describe } \\
\text { the implementation } \\
\text { process in different } \\
\text { contexts and evaluate } \\
\text { fidelity }\end{array}$ \\
$\begin{array}{l}\text { B: Primary } \\
\text { health care } \\
\text { centers }\end{array}$ & $\begin{array}{l}\text { and implementation } \\
\text { process will be guided } \\
\text { by the RIV } \\
\text { specification for SCP } \\
\text { (Boxes 1 and } 2)\end{array}$ & $\begin{array}{l}\text { Effect of SCP: } \\
\text { How SCP affects the } \\
\text { quality of } \\
\text { documentation and } \\
\text { the use of } \\
\text { evidence-based care }\end{array}$ \\
\hline
\end{tabular}

PHCC, the project group will be formed by nurses. In these groups, one person with competence in informatics will be included as an external facilitator. The work of the group will be to develop an SCP according to the steps in the RIV specification [10] (Box 1).

The group will meet continually and the time to complete the mission will depend on the resources. IJ and ET will be included in the groups as mentors and will simultaneously act as researchers to study the process. The manager of the organization and medically responsible physicians will approve the SCP. To implement the SCP developed, it has to be integrated into the electronic patient record. The person with competence in informatics will be needed for this part of the development of the SCP.

To implement the SCP in everyday work on the ward and at the PHCC, internal facilitators will be used. IJ and ET will support the facilitators during the introduction and education. The facilitators will introduce and educate the staff on how to use the SCP according to the steps in the RIV specification [10] (Box 2). Before the implementation of SCP can begin, the introduction and approach must be thoroughly anchored at the manager. It appeared that the motivation for nurses to use the SCP was that it was easy to understand, consistent with applicable standards and regulations, and that the leadership had given their approval and that there was a clear division of roles in the implementation [15]. The facilitators will continue to support the teams to use SCP in their daily work, and evaluate the applicability of the SCP.

Beyond those two known mechanisms, several others mechanisms will work as triggers for fidelity and

Development of an SCP:

- Establish a project group

- Make an inventory of available SCPs

- Decide if a new SCP shall be developed or an available SCP shall be used

- Identify and state which knowledge base shall be used

- $\quad$ Create an SCP

- Assure the quality of the content

- Approve the SCP

- $\quad$ Register the SCP

Box 1. Development of an SCP according to the steps in the RIV specification [10].

Implementation of an SCP

- Search for an SCP

- Assess/motivate/decide on an SCP

- Bring the SCP to the individual care plan

- Perform care according to the applied SCP

- Evaluate the applicability of the SCP

Box 2. Implementation of an SCP according to the steps in the RIV specification [10]. 
outcomes $[25,26]$. These mechanisms will be investigated and as far as possible explained by using action research, described later in this article. The result of the investigation will describe the relationship between CMO rather than explain the causality [23].

The outcome $(\mathrm{O})$ of the study will be studied in two phases: phase 1 will investigate the development and implementation process by evaluating fidelity; and phase 2 will evaluate how the SCP affects the quality of documentation and the use of evidence-based care.

\subsection{Phase 1: Investigation of the Implementation Process}

In realistic evaluation, mixed methods can be used $[21,22]$. To study the implementation process, action research will be used. Action research can be defined as: "The study of social situations carried out by those involved in that situation in order to improve their practice and the quality of their understanding” [27, p. 8]. In action research, the process starts by getting a group of people together who would like to do the work for their own purposes based on their experience. The focus will be twofold for the participants and the researcher: on the actions in the clinic and their own learning. These foci will be the source of different action-reflection cycles [28].

In the first cycle of the process, an SCP will be developed and implemented in the electronic patient record, Box 1. This will give an action-reflection cycle in which new knowledge obtained while developing the SCP may lead to changes in the care performed. In the second cycle, the SCP will be implemented in the everyday work on the ward, in the two parts in the RIV specification described earlier, Box 2. Implementing the SCP in daily practice will provide knowledge about the attitudes and behavior of the personnel when changes take place.

To study the implementation process, focus groups interviews will take place with the participating staff members and managers. These interviews will be recorded and transcribed before analysis. The implementation process will also be observed by the researcher using field notes and log books for the personnel responsible.

All personnel employed in the two care setting will also be invited to participate in an inquiry dealing with the prerequisites for implementing a specific routine, using a questionnaire originally developed for measuring the factors and prerequisites for implementing clinical guidelines on the basis of the Promoting Action on Research Implementation in Health Service (PARIHS) framework [29,30]. This inquiry will be repeated at the end of the project. After the implementation, the application of the SCP will be examined by auditing patient records.

The qualitative data from the focus groups interviews will be analyzed using Grounded Theory [31], a method often used to study social processes when action research is used. The quantitative data from the questionnaire will be analyzed by descriptive statistics in SPSS. Comparisons will be done between the two contexts.

\subsection{Phase 2: Evaluation of the Effects of Using SCP}

Examination of double documentation and adherence to evidence-based care in the electronic patient record will be done before and after the SCP is implemented. Electronic patient record will be collected during a 3-month period before the start of the project and for a 3-month period 1 year after the SCP has been implemented in the electronic record. Approximately 90 records from the orthopedic clinic and about 10 - 30 records per PHCC will be included depending on the patient group (i.e. patients with a leg ulcer or hypertension). A study-specific audit protocol appropriate for each patient group in which a SCP is created will be used. The audit protocol will be constructed by the authors based on the knowledge base for the SCP. The data collected from the electronic patient record will be analyzed using summative content analysis [32].

Focus group interviews will also take place before implementation and 6 - 12 months after implementation to evaluate the personnel's experience of the usefulness of the information in the patient records and the effects of the SCP in their work after implementation; the interviews will be analyzed using conventional content analysis [32]. Comparisons will be done between the two contexts.

A summary description of the process, context, and planned studies for implementation of SCPs is given in Figure 1.

\subsection{Ethical Considerations}

To develop and implement SCPs should be part of the legal obligation of health care personnel to continuously update their knowledge and to transfer important information about the patient. Implementation of SCPs will not influence the possibility of the patient getting the right care at the right time, rather it will optimize the care given. No changes to or new methods of care and treatment will take place that are not verified by research or evidence-based knowledge.

The World Medical Association's Declaration of Helsinki [33] and the Swedish Act on Ethical Review of Research Involving Humans [34] were taken into account for the study. Permission to carry out the study has been granted by a Regional Ethics Committee (Ref: 2010/670 and Ö 9-2011).

The participants will receive verbal and/or written in- 


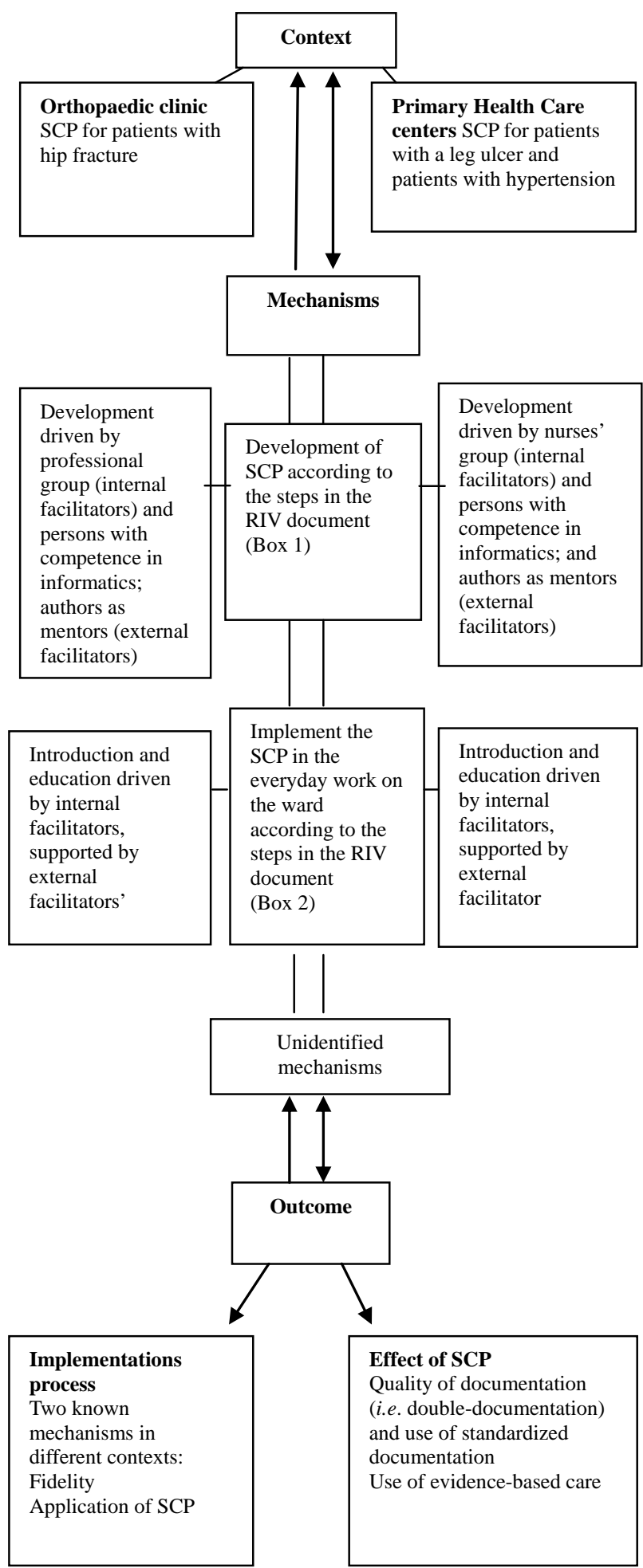

Figure 1. Summary description of the process, context, and planned studies for implementation of SCPs.

formation, and their informed written consent will be obtained before participation. Participation will be voluntary and the informants will be told that they can withdraw from the study at any time. Confidentiality will be guaranteed.

\section{DISCUSSION}

This article presents the theoretical and methodological considerations for designing a research study to evaluate the implementation of an SCP. Efforts to develop an SCP are a challenge because development means combining the views of multiple health professionals and accomplishing a joint health care agreement. Implementation of the SCP will probably meet the same resistance as implementation of guidelines [7,35]. Most health professionals know what guidelines are valid and should be used but then do not use them; colleagues and experiences are used instead. Physicians should discuss with each other how to tackle the discrepancy between guidelines and practice. Nurses use guidelines for an unknown problem but when they have learned how to solve the problem, they no longer use the guidelines [35]. A systematic evaluation of the implementation process considering the various and changing contexts of health care and the mechanisms embedded in social processes could provide one more piece in the puzzle of what makes an implementation successful.

Because documentation of care is an important but resource-consuming requirement in health care, a more standardized method of documenting is requested by health professionals. This project can provide insight into the complex process of developing and implementing an SCP in different contexts, which will be useful in further implementation processes. It could also increase our understanding of the barriers and possibilities in creating a generic model for interdisciplinary documentation of care, and evaluate the RIV specification [10] that will guide the process.

\section{REFERENCES}

[1] Estabrooks, C.A., Floyd, J.A., O’Leary, K.A. and Gushta, M. (2003) Individual determinants of research utilization: a systematic review. Journal of Advanced Nursing, 43, 506-520. http://dx.doi.org/10.1046/j.1365-2648.2003.02748.x

[2] Carlson, C.L. and Plonczynski, J. (2008) Has the BARRIERS Scale changed nursing practice? Journal of Advanced Nursing, 63, 322-333.

http://dx.doi.org/10.1111/j.1365-2648.2008.04705.x

[3] Squires, J.E., Hutcinson, A.M., Boström, A.-M., O’Rouke, H.M., Cobban, S.J. and Estabrooks, C.A. (2011) To what extent do nurses use research in clinical practice? A systematic review. Implementation Science, 6, 21. http://dx.doi.org/10.1186/1748-5908-6-21

[4] Thomas, L.H., Cullum, N.A., McColl, E., Rousseau, N. and Steen, N. (2000) Guidelines in professions allied to medicine (review). The Cochrane Library. http://dx.doi.org/10.1002/14651858.CD000349 
[5] Natsch, S. and van der Meer, J.W.M. (2003) The role of clinical guidelines, policies and stewardship. Journal of Hospital Infection, 53, 172-176. http://dx.doi.org/10.1053/jhin.2002.1372

[6] Gooch, P. and Roudsari, A. (2011) Computerization of workflows, guidelines, and care pathways: A review of implementation challenges for process-oriented health information systems. Journal of the American Medical Informatics Association, 18, 738-748.

http://dx.doi.org/10.1136/amiajnl-2010-000033

[7] Grol, R. and Grimshaw, J. (2003) From best evidence to best practice: Effective implementation of change in patients' care. Lancet, 362, 1225-1230.

http://dx.doi.org/10.1016/S0140-6736(03)14546-1

[8] Conrad, D. and Stocker Sneider, J. (2011) Enhancing the visibility of NP practice in electronic health records. The Journal for Nurse Practitioners, 7, 832-838. http://dx.doi.org/10.1016/j.nurpra.2011.04.004

[9] Wang, N., Hailey, D. and Yu, P. (2011) Quality of nursing documentation and approaches to its evaluation: A mixed-method systematic review. Journal of Advanced Nursing, 67, 1858-1875.

http://dx.doi.org/10.1111/j.1365-2648.2011.05634.x

[10] RIV (2011) RIV-Specifikation Nationell informationsstruktur för standardiserade vårdplaner [RIV specification -National information infrastructure for standardized care plans].

http://www.cehis.se/images/uploads/dokumentarkiv/RIV specifikation nat inform struktur fr SVP 110610.pdf

[11] De Bleser, L., Depreitere, R., de Waele, K., Vanhaecht, K., Vlayen, J. and Sermeus, W. (2006) Defining Pathways. Journal of Nursing Management, 14, 553-563. http://dx.doi.org/10.1111/j.1365-2934.2006.00702.x

[12] Kinsman, L., Rotter, T., James, E., Snow, P. and Willis, J. (2010) What is clinical pathway? Development of a definition to inform the debate. BMC Medicine, 8, 31. http://dx.doi.org/10.1186/1741-7015-8-31

[13] Social Department (2010) Nationella eHälso-strategin [National strategy for eHealth]. http://www.regeringen.se/sb/d/14914/a/148429

[14] Dahm, M.F. and Wadensten, B. (2008) Nurses' experiences of and opinions about using standardized care plans in electronic health records-A questionnaire study. Journal of Clinical Nursing, 17, 2137-2145. http://dx.doi.org/10.1111/j.1365-2702.2008.02377.x

[15] Jansson, I., Bahtsevani, C., Pilhammar, E. and Forsberg, A. (2010) Factors and conditions that influence the implementation of standardized nursing care plans. Open Journal of Nursing, 4, 25-34. http://dx.doi.org/10.2174/1874434601004010025

[16] Lee, T.-T. and Chang, P.-C. (2004) Standardized care plans: Experiences of nurses in Taiwan. Journal of Clinical Nursing, 13, 33-40. http://dx.doi.org/10.1111/j.1365-2702.2004.00818.x

[17] Pöder, U., Fogelberg-Dahm, M. and Wadensten, B. (2011) Implementation of a multi-professional standardized careplan in electronic health records for the care of stroke patients. Journal of Nursing Management, 19, 810-819. http://dx.doi.org/10.1111/j.1365-2834.2011.01220.x
[18] Vanhaecht, K., De Witte, K., Panelle, M. and Sermeus, W. (2009) Do pathways lead to better organized care processes? Journal of Evaluation in Clinical Practice, 15, 782-788.

http://dx.doi.org/10.1111/j.1365-2753.2008.01068.x

[19] Hunter, B. and Segrott, J. (2008) Re-mapping client journeys and professional identities: A review of the literature on clinical pathways. International Journal of Nursing Studies, 45, 608-635. http://dx.doi.org/10.1016/j.ijnurstu.2007.04.001

[20] Matland, R.E. (1995) Synthesizing the implementation literature: The ambiguity-conflict model of policy implementation. J-PART, 5, 145-174.

[21] Pawson, R. and Tilly, N. (1997) Realistic Evaluation. Sage Publications, London.

[22] Pommier, J., Guével, M.-R. and Jourdan, D. (2010) Evaluation of health promotion in schools: A realistic evaluation approach using mixed methods. BMC Public Health, 10, 43. http://dx.doi.org/10.1186/1471-2458-10-43

[23] Wilson, V. and McCormack, B. (2006) Critical realism as emancipatory action: The case for realistic evaluation in practice development. Nursing Philosophy, 7, 45-57. http://dx.doi.org/10.1111/j.1466-769X.2006.00248.x

[24] Wand, T., White, K. and Patching, J. (2011) Realistic evaluation of an emergency department-based mental health nurse practitioner outpatient service in Australia. Nursing \& Health Sciences, 13, 199-206. http://dx.doi.org/10.1111/j.1442-2018.2011.00601.x

[25] Carroll, C., Patterson, M., Wood, S., Booth, A., Rick, J. and Balain, S. (2007) A conceptual framework for implementation fidelity. Implementation Science, 2, 40.

[26] Williams, L., Burton, C. and Rycroft-Malone, J. (2012) What works: A realist evaluation case study of intermediaries in infection control practice. Journal of Advanced Nursing, 69, 915-926. http://dx.doi.org/10.1111/j.1365-2648.2012.06084.x

[27] Winter, R. and Munn-Giddings, C. (2001) A Handbook for Action Research in Health and Social Care. Routledge, London.

[28] McNiff, J. and Whitehead, J. (2011) All You Need to Know About Action Research. 2nd Edition, Sage Publications, London.

[29] Rycroft-Malone, J., Kitson, A., Harvey, G., McCormack, B., Seers, K., Tichen, A. and Eastbrooks, C. (2002) Ingredients for changes: Revisting a conceptual model. Quality and Safety in Health Care, 11, 174-180. http://dx.doi.org/10.1136/qhc.11.2.174

[30] Bahtsevani, C., Khalaf, A., Willman, A. and Östman, M. (2008) Developing an instrument for evaluating implementation of clinical practice guidelines: A test-retest study. Journal of Evaluation in Clinical Practice, 14, 839-846. http://dx.doi.org/10.1111/j.1365-2753.2007.00916.x

[31] Charmaz, K. (2005) Grounded theory in the 21st century. In: Denzin, N.K. and Lincoln, Y.S., Eds., Handbook of Qualitative Research, The Sage, London, Chapter 20.

[32] Hsieh, H.-F. and Shannon, S.E. (2005) Three approaches to qualitative content analysis. Qualitative Health Re- 
search, 15, 1277-1288.

http://dx.doi.org/10.1177/1049732305276687

[33] World Medical Association (2003) Declaration of Helsinki. In The Swedish Research Council's Guidelines for the Ethical Evaluation of Medical Research on Humans. Medicinska forskningsrådet (MFR), Stockholm, 99-104.

[34] SFS (2003) Lag om etikprövning av forskning som avser människor [Act on the Ethical Review of Research
Involving Humans]. Socialdepartementet. Stockholm, 460.

[35] Gabbay, J. and le May, A. (2004) Evidence based guidelines or collectively constructed "mindlines"? Ethnographic study of knowledge management in primary care. BMJ, 329, 1013.

http://dx.doi.org/10.1136/bmj.329.7473.1013 